\title{
ON A THEOREM OF MORI AND THE DEFINITION OF QUASICONFORMALITY $\left({ }^{1}\right)$
}

\author{
BY \\ LIPMAN BERS
}

A posthumous paper by the late Professor Akira Mori [15] contains implicitly a solution of an important problem in the theory of quasiconformal mappings. More precisely, using Mori's Theorem I we can show, in a few lines, that two generally accepted "natural" definitions of quasiconformality are equivalent. In order to make this note readable, however, we shall need more than a few lines for a restatement of these definitions.

1. Grötzsch's inequality. The concept of quasiconformality is due to Grötzsch $[10 ; 11 ; 12]$ who considered primarily homeomorphisms

$$
w(z)=u(x, y)+i v(x, y) \quad(z=x+i y)
$$

of class $C^{1}$ with a positive Jacobian

$$
J=u_{x} v_{y}-u_{y} v_{x} .
$$

Such a mapping takes infinitesimal circles into infinitesimal ellipses; it is called quasiconformal if the eccentricity of these ellipses is uniformly bounded. This condition can be expressed analytically by either of the three equivalent differential inequalities:

$$
\begin{aligned}
& \max _{0 \leqq \theta \leqq 2 \pi}\left|w_{x} \cos \theta+w_{y} \sin \theta\right|^{2} \leqq Q J, \\
& u_{x}^{2}+u_{y}^{2}+v_{x}^{2}+v_{y}^{2} \leqq\left(Q+\frac{1}{Q}\right) J, \\
& \left|w_{x}+i w_{y}\right| \leqq \frac{Q-1}{Q+1}\left|w_{x}-i w_{y}\right|,
\end{aligned}
$$

for some $Q \geqq 1$. This property is conformally invariant: if $w=w(z)$ has it, so does the function $W(\zeta)=F\{w[f(\zeta)]\}$ where $F$ and $f$ are conformal mappings.

Consider, in particular, a quasiconformal mapping, with constant $Q$, of the closed rectangle $0 \leqq x \leqq a, 0 \leqq y \leqq b$ onto another closed rectangle $0 \leqq u \leqq a^{\prime}$, $0 \leqq v \leqq b^{\prime}$ assuming that the vertices $(0,0),(0, a),(a, b),(0, b)$ are taken into $(0,0),\left(0, a^{\prime}\right),\left(a^{\prime}, b^{\prime}\right),\left(0, b^{\prime}\right)$ respectively. A simple application of the Schwartz inequality (cf. Ahlfors [1]) yields the Grötzsch inequality

Received by the editors August 23, 1956.

(1) Work supported under contract DA-30-069-ORD-835 with the Office of Ordnance Research, United States Army. Reproduction in whole or in part permitted for any purpose of the United States Government. 


$$
\frac{a^{\prime}}{b^{\prime}} \leqq Q \frac{a}{b}
$$

More generally, let $w$ be a quasiconformal homeomorphism of a domain $D$ onto a domain $\Delta$ and let $R \subset D$ be a topological rectangle (topological image of the closed square with vertices $\alpha_{1}=(0,0), \alpha_{2}=(1,0), \alpha_{3}=(1,1), \alpha_{4}=(0,1)$. $R$ can be mapped conformally onto the rectangle $0 \leqq x \leqq a, 0 \leqq y \leqq b$ in such a way that the points $\alpha_{1}, \alpha_{2}, \alpha_{3}, \alpha_{4}$ go into the points $(0,0),(a, 0),(a, b)$, $(0, b)$, respectively, and the uniquely determined number $(a / b)$ is called the modulus of $R$ and is denoted by $\bmod R$. In view of (2) and the conformal invariance of inequality (1) it follows that

$$
\bmod R \leqq Q \bmod w(R) .
$$

Note that topological rectangles and their moduli may be considered on arbitrary Riemann surfaces.

2. Geometric definition. Quasiconformal mappings have proved to be a powerful tool in the theory of functions (cf. Ahlfors [1], Pfluger [18], Volkoviskii [23], Cacciopoli [6] and the references given there), especially in connection with Teichmüller's extremal quasiconformal mappings [22], and in the theory of partial differential equations (cf., in particular, Morrey [16], Lavrent'ev [13;14], Bers [2; 3], Nirenberg [17], Finn [8], Bers and Nirenberg [4]). But in applying this tool it became necessary to extend the original definition. Of the proposed generalizations two are, in a certain sense, most general.

The geometric definition (Ahlfors, Pfluger, Mori) dispenses with all differentiability requirements and uses directly inequality (3). According to this definition a homeomorphism $w$ of a plane domain $D$ onto another such domain or, more generally, of a Riemann surface $D$ onto another such surface, is $Q$-quasiconformal if (3) holds for every topological rectangle $R \subset D$.

Consider now an interior function $w(z)$ defined in a domain $D$. This means that $w(z)$ is continuous and either constant or has the following three properties. (i) The mapping $w$ is light and open $\left({ }^{2}\right)$. (ii) In the neighborhood of every point of $D$, save perhaps for a discrete set, the mapping $w$ is a local homeomorphism. (iii) There exists a homeomorphism $\chi(z)$ of $D$ onto a plane domain and an analytic function $f(\zeta)$ defined in $\chi(D)$ such that

$$
w(z)=f[\chi(z)] .
$$

The three properties (i), (ii), (iii) are equivalent. The implications (iii) $\rightarrow$ (i) and (iii) $\rightarrow$ (ii) are trivial. The implication (i) $\rightarrow$ (iii) is the well known result of Stoilow [20]. The implication (ii) $\rightarrow$ (iii) is an easy consequence of the general uniformization theorem $\left({ }^{3}\right)$. A nonconstant interior function $w$ may be

(2) Cf. Whyburn [24].

(3) Cf. [3, p. 454]. 
considered as a homeomorphism of $D$ onto a Riemann covering surface of a plane domain. Mori calls $w$ pseudoanalytic if this homeomorphism is quasiconformal.

We shall say "quasiconformal" rather than "pseudoanalytic" $\left.{ }^{4}\right)$ and shall call a function $w, Q$-quasiconformal according to the geometric definition if it is of the form (4) where $\chi$ is a $Q$-quasiconformal homeomorphism and $f$ an analytic function. Mori himself noted that this definition is equivalent to his.

3. Analytic definition. We recall the concept of $L_{2}$ derivatives due to Sobolev [19] and Friedrichs [9]. Let $f, g, h$ be measurable, locally square integrable, complex or real valued functions defined in a plane domain $D$. The relations

$$
g=f_{x}, \quad h=f_{y}
$$

in the $L_{2}$ sense

mean that the following conditions are satisfied. (a) The identities

$$
\iint_{D} f \omega_{x} d x d y=-\iint_{D} g \omega d x d y, \quad \iint_{D} f \omega_{y} d x d y=-\iint_{D} h \omega d x d y
$$

hold for every function $\omega$ of class $C^{1}$ with compact support $S \subset D$. (b) In every compact set $S \subset D$ there exist functions $f^{(n)}$ of class $C^{1}$ such that

$$
\iint_{S}\left\{\left|f^{(n)}-f\right|^{2}+\left|f_{x}^{(n)}-g\right|^{2}+\left|f_{y}^{(n)}-h\right|^{2}\right\} d x d y \rightarrow 0
$$

as $n \rightarrow \infty$. (c) The function $f(x, y)$ is absolutely continuous in $x$ for almost all values of $y$ and in $y$ for almost all values of $x$, and $f_{x}=g, f_{y}=h$ almost everywhere in $D$.

It is known that each of the properties (a), (b), (c) implies the other two.

According to the analytic definition (Morrey, Cacciopoli, Bers and Nirenberg) a continuous function $w(z)$ in a domain $D$ is $Q$-quasiconformal if it has $L_{2}$ derivatives satisfying inequality (1) almost everywhere.

4. Beltrami equations. Let

$$
g_{11}(x, y) d x^{2}+2 g_{12}(x, y) d x d y+g_{22}(x, y) d y^{2}
$$

be a Riemann metric defined in a domain $D$. A function $w=u+i v$ in $D$ is said to be conformal with respect to this metric if $u$ and $v$ satisfy the Beltrami equations

$$
g u_{x}=-g_{12} v_{x}+g_{11} v_{y}, \quad g u_{y}=-g_{22} v_{x}+g_{12} v_{y}
$$

where

$$
g^{2}=g_{11} g_{22}-g_{12}^{2}
$$

(4) In the writer's opinion the term "pseudoanalytic" should be reserved for solutions of generalized Cauchy-Riemann equations. 
which can be also written in the form

$$
w_{x}+i w_{y}=\mu\left(w_{x}-i w_{y}\right),
$$

the complex valued function $\mu$ being given by

$$
\mu=\frac{g_{11}-g_{22}+2 i g_{12}}{g_{11}+g_{22}+2 g} .
$$

In what follows we assume that the functions $g_{i k}$ are measurable and that the eccentricity of the metric is bounded by $Q \geqq 1$, that is, that

$$
g_{11}+g_{22} \leqq 2 Q g
$$

or, which is the same,

$$
|\mu|<(Q-1) /(Q+1) .
$$

A solution of (6) will be required to be continuous and to have $L_{2}$ derivatives satisfying the equation almost everywhere. Thus every solution of a Beltrami system is $Q$ quasiconformal (by the analytic definition) if the eccentricity of the metric is bounded by $Q$, and conversely, every quasiconformal function (according to the analytic definition) is a solution of an appropriately chosen Beltrami sysțem.

We note now some properties of a Beltrami system (6) satisfying (7).

$(\alpha)$ If $w_{1}$ and $w_{2}$ are two solutions of (6) in the same domain and $w_{1}$ is a homeomorphism, $w_{2}$ is an analytic function of $w_{2}$.

$(\beta)$ If $w$ is a solution of (6) so is $f(w), f$ being any analytic function.

$(\gamma)$ In every domain there exists a homeomorphism satisfying (6).

( $\delta$ ) If $w=u+i v$ is a univalent solution of (6) in a domain $D$, the Jacobian $J=u_{x} v_{y}-u_{y} v_{x}$ is positive almost everywhere, and for every measurable set $e \subset D, w(e)$ is measurable and has measure

$$
\iint_{D} J d x d y \text {. }
$$

These results are due to Morrey [16]. For different proofs cf. Bers and Nirenberg [4] and Boyarskir [5]. Boyarskir's proof is based on the CalderónZygmund inequality [7]; it implies that the moduli of the derivatives of a solution of (6) are locally integrable to a power $p>2$, depending only on $Q$.

It can be shown that every measurable locally square integrable function having $L_{2}$ derivatives satisfying (6) is continuous. Hence the continuity hypothesis may be omitted from the analytic definition of quasiconformality.

We shall show next that the two definitions are equivalent. Hence statement $(\delta)$ answers in the affirmative a question raised by Mori $[15, \S 5]$.

5. Equivalence proof. A homeomorphism which is $Q$-quasiconformal according to the analytic definition is so also according to the geometric definition. In fact, noting statement $(\delta)$ the usual proof of Grötzsch's inequality 
can be repeated almost verbatim. Statement $(\alpha)$ now leads to the (known) observation that the analytic definition implies the geometric.

Now let $w(z)=u+i v$ be a homeomorphism of a domain $D$ which is $Q$ quasiconformal according to the geometric definition. Mori proved (Theorem I) that $w(x+i y)$ is absolutely continuous on almost all lines $x=$ const. and $y=$ const. $\left(^{5}\right)$, and that there exists a null set $E \subset D$ such that at every point of $D-E, w$ has a differential and its derivatives satisfy (1). In order to verify that $w$ is a solution of a Beltrami system we must only show that its derivatives are locally square integrable.

For every Borel set $e \subset D$ let $s(e)$ denote the measure of the Borel set $w(e)$; $s(e)$ is a countably additive non-negative set function. Let $\Delta\left(z_{0}, r\right)$ denote the disc $\left|z-z_{0}\right|<r$. It is well known that the Lebesgue derivative

$$
h\left(z_{0}\right)=\lim _{r \rightarrow 0} \frac{s\left[\Delta\left(z_{0}, r\right)\right]}{\pi r^{2}}
$$

exists almost everywhere in $D$, and

$$
\iint_{\theta} h d x d y \leqq s(e) \text {. }
$$

Mori observed, and it is easy to verify, that

$$
h=J=u_{x} v_{y}-u_{y} v_{x}
$$

at all points of $D-E$. Hence, for every compact set $S \subset D$ we have by (1) and (8) that

$$
\begin{aligned}
\iint_{S}\left(u_{x}^{2}+u_{y}^{2}+v_{x}^{2}+v_{y}^{2}\right) d x d y & \leqq(Q+1 / Q) \iint_{S} J d x d y \\
& \leqq(Q+1 / Q) s(S)<+\infty .
\end{aligned}
$$

Noting statement $(\beta)$ of $\S 4$ we conclude that the geometric definition of $Q$-quasiconformality implies the analytic definition.

6. An application. Mori asked $[15, \S 5]$ whether $Q$-quasiconformal functions of class $C^{1}$ are dense in the set of all $Q$-quasiconformal functions, in the sense of normal convergence (uniform convergence on compact subsets). The equivalence theorem yields the affirmative answer.

Let $w(z)$ be a $Q$-quasiconformal function; for the sake of simplicity we assume it to be defined in a subdomain $D$ of the unit disc $|z|<1$. $w$ is a solution of (6) with some $\mu$ satisfying $|\mu|<(Q-1) /(Q+1)$. We may assume that $\mu$ is defined for $|z|<1$.

$\mathrm{By}(\gamma)$ there exists a homeomorphic solution $W_{0}(z)$ of $(6)$ defined in $|z|<1$. Since quasiconformal mappings are known to preserve conformal type the image $\Delta$ of $|z|<1$ under $W_{0}$ is not the whole plane. Let $F(\zeta)$ be the analytic

(5) This was also proved, independently, by Strebel [21]. 
function mapping $\Delta$ conformally onto the unit disc, $F\left[W_{0}(0)\right]=0$. The function $W(z)=F\left[W_{0}(z)\right]$ is a solution of (6) which maps $|z|<1$ homeomorphically onto itself and leaves the origin fixed.

By a fundamental property of quasiconformal mapping (discovered by Morrey, Ahfors and Lavrent'ev and, in its sharp form, by Mori)

$$
\left(\left|z_{1}-z_{2}\right| / 16\right)^{Q} \leqq\left|W\left(z_{1}\right)-W\left(z_{2}\right)\right| \leqq 16\left|z_{1}-z_{2}\right|^{1 / Q} \text {. }
$$

$W$ is a homeomorphism of $|z| \leqq 1$ and we may assume that $W(1)=1$, since this can be achieved by a rotation.

Now let $\left\{\mu^{(n)}\right\}$ be a sequence of complex valued real-analytic functions such that

$$
\begin{aligned}
\left|\mu^{(n)}\right| & \leqq(Q-1) /(Q+1), \\
\mu^{(n)} & \rightarrow \mu \text { a.e. in }|z|<1 .
\end{aligned}
$$

For every $n$ there exists a solution $W^{(n)}(z)$ of the equation

$$
W_{x}^{(n)}+i W_{y}^{(n)}=\mu^{(n)}\left(W_{x}^{(n)}-i W_{y}^{(n)}\right)
$$

which is a homeomorphism of $|z| \leqq 1$ onto itself satisfying the conditions $W^{(n)}(0)=0, W^{(n)}(1)=1$. These functions are real analytic and each satisfies inequalities (9). Hence we may assume, selecting if need be a subsequence, that $W^{(n)}(z)$ converges uniformly to a homeomorphism $W^{(\infty)}(z)$ of $|z| \leqq 1$ onto itself. Since, by (1),

$$
\iint_{D}\left\{\left|W_{x}^{(n)}\right|^{2}+\left|W_{y}^{(n)}\right|^{2}\right\} d x d y \leqq(Q+1 / Q) \pi
$$

we may assume, selecting if need be a subsequence, that the functions $W_{x}^{(n)}$, $W_{y}^{(n)}$ converge weakly to certain $L_{2}$ functions which are easily seen to be the $L_{2}$ derivatives of $W^{(\infty)}$. It follows that $\mu^{(n)}\left\{W_{x}^{(n)}-i W_{y}^{(n)}\right\}$ converges weakly to $\mu\left(W_{x}^{(\infty)}-i W_{y}^{(\infty)}\right)$, so that $W^{(\infty)}$ is a solution of $(6)$. By $\S 4,(\alpha)$ we have that $W^{(\infty)}(z)=F[W(z)], F$ being analytic. But $F=W^{(n)} \circ W^{-1}$ is a homeomorphism of $|z| \leqq 1$ onto itself leaving 0 and 1 fixed. Hence $W^{(\infty)}=W$ and $W^{(n)} \rightarrow W$ uniformly.

Next, $w(z)$ must be of the form $w(z)=f[W(z)], f$ being analytic in $W(D)$. Let $S$ be any subdomain of $D$ with compact closure $\bar{S} \subset D$. If $n$ is sufficiently large, $W^{(n)}(\bar{S}) \subset W(D)$. The function $w^{(n)}(z)=f\left[W^{(n)}(z)\right]$ is $Q$-quasiconformal in $S$ and is of class $C^{1}$, in fact real analytic there. Clearly $w^{(n)} \rightarrow w$ uniformly in $S$.

There are several obvious modifications of the preceding argument. These may be left to the reader.

\section{BIBLIOGRAPHY}

1. L. Ahlfors, On quasi-conformal mappings, Journal d'Analyse Mathématique vol. 4 (1954) pp. 1-58. 
2. L. Bers, Univalent solutions of linear elliptic systems, Comm. Pure Appl. Math. vol. 6 (1953) pp. 513-526.

3. —- Existence and uniqueness of a subsonic flow past a given profile, Ibid. vol. 6 (1954) 441-504.

4. L. Bers and L. Nirenberg, On a representation theorem for linear elliptic systems with discontinuous coefficients and its applications, Convegno Internazionale sulle Equazioni Derivate e Parziali, Agosto, 1954, pp. 111-140.

5. B. V. Boyarskil, Homeomorphic solutions of Beltrami systems, Doklady Akademii Nauk SSSR. vol. 102 (1955) pp. 661-664.

6. R. Caccioppoli, Fondamenti per una teoria generale delle funzioni pseudo-analitiche di una variabile complessa, Accademia Nazionale dei Lincei, Rend. vol. 13(1952) pp. 197-204 and pp. 321-351.

7. H. P. Calderon and A. Zygmund, On the existence of certain singular integrals, Acta Math. vol. 88 (1952) pp. 85-139.

8. R. S. Finn, On a problem of type, with application to elliptic partial differential equations, Journal of Rational Mechanics and Analysis vol. 3 (1954) pp. 789-799.

9. K. O. Friedrichs, The identity of weak and strong extensions of differential operators, Trans. Amer. Math. Soc. vol. 55 (1944) pp. 132-151.

10. H. Grötzsch, Ueber die Verzerrung bei schlichten nichtkonformen Abbildungen und über eine damit zusammenhängende Erweiterung des Picardschen Satzes, Leipzig-Berlin, vol. 80, 1928.

11. - Ueber die Verzerrung bei nichtkonformen schlichten Abbildungen mehrfach zusammenhängender Bereiche, Leipzig-Berlin, vol. 82, 1930.

12. ——, Ueber möglichst konforme Abbildungen von schlichten Bereichen, Leipzig-Berlin, vol. $84,1932$.

13. M. A. Lavrent'ev, A general problem of the theory of quasi-conformal representation of plane regions, Rec. Math. (Mat. Sbornik) N.S. vol. 21 (1947) pp. 285-326.

14. - A fundamental theorem of the theory of quasi-conformal mapping of plane regions, Izvestiya Akademii Nauk SSSR. vol. 12 (1948) pp. 513-554.

15. A. Mori, On quasi-conformality and pseudo-analyticity, Trans. Amer. Math. Soc. vol. 68 (1957) pp. 56-77.

16. C. B. Morrey, On the solution of quasilinear elliptic partial differential equations, Trans. Amer. Math. Soc. vol. 43 (1938) pp. 126-166.

17. L. Nirenberg, On nonlinear elliptic partial differential equations and Hölder continuity, Comm. Pure Appl. Math. vol. 6 (1953) pp. 97-156.

18. A. Pfluger, Quasikonforme Abbildungen und logarithmische Kapazität, Ann. Inst. Fourier vol. 2 (1950) pp. 69-80.

19. S. L. Sobolev, Some applications of functional analysis to mathematical physics, Leningrad Univ., Leningrad, 1950 (Russian).

20. S. Stoīlow, Leçons sur les principes topologiques de la théorie des fonctions analytiques, Paris, Gauthier-Villars, 1938.

21. K. Strebel, On the maximum dilation of quasi-conformal mappings, Bull. Amer. Math. Soc. vol. 63 (1955) p. 225.

22. O. Teichmuller, Untersuchungen über konforme und quasikonforme Abbildungen, Deutsche Mathematik vol. 3 (1938) pp. 621-678.

23. L. I. Volkoviskii, Investigation on the problem of type of a simply-connected Riemann surface, Moscow, Izdat. Akad. SSSR, 1950. (Akad. Nauk Trudy Matem. Inst. imeni V. A. Steklova, no. 34.)

24. G. T. Whyburn, Introductory topological analysis, Lectures on Functions of a Complex Variable, Ann Arbor, The University of Michigan Press, 1955.

New York UNIVERSITY,

NEW YORK, N. Y. 